\title{
Clinical benefits and costs of an outpatient parenteral antimicrobial therapy service.
}

\author{
BUGEJA, S.J., STEWART, D. and VOSPER, H.
}


Clinical benefits and costs of an Outpatient Parenteral Antimicrobial Therapy service

\section{Dr. Sara Jo Bugeja}

Robert Gordon University, School of Pharmacy and Life Sciences, Sir lan Wood Building, Garthdee Road, Aberdeen, AB10 9GJ, UK

\section{Prof. Derek Stewart}

College of Pharmacy, QU Health, Qatar University, Doha, Qatar

\section{Dr. Helen Vosper (corresponding author)}

Robert Gordon University, School of Pharmacy and Life Sciences, Sir lan Wood Building, Garthdee Road, Aberdeen, AB10 9GJ, UK 


\section{Introduction}

Background to outpatient parenteral antimicrobial therapy (OPAT)

Outpatient parenteral antimicrobial therapy (OPAT) provision is described as "the administration of parenteral antimicrobial therapy in at least two doses on different days without intervening hospitalization"1 ${ }^{1}$. OPAT was first documented by Rucker and Harrison in describing the successful management of a paediatric cohort $^{2}$. OPAT services have since expanded globally, with practices evolving in the management of a wide range of patient indications involving numerous antimicrobial agents, vascular devices and administration procedures ${ }^{3,4}$. There are three main OPAT models: the home model (which may involve either selfadministration or nurse administration), with the other two models requiring the patient to return to a clinical setting of either a skilled nursing facility or the infusion centre ${ }^{5}$. As OPAT has gained traction worldwide, there has been an accumulation of evidence, centring on robust outcome measures of clinical effectiveness, safety, economic gain and patient satisfaction, with benefits for patients, practitioners and healthcare organisations $s^{6,7}$. This evidence has translated into a myriad of guidelines, including those recently issued by the Infectious Diseases Society of America (IDSA) ${ }^{1}$ and the British Society for Antimicrobial Chemotherapy (BSAC) ${ }^{8}$. 
The national OPAT service in Malta

The Maltese national acute facility, Mater Dei Hospital (MDH), extends its services free of charge to all those patients who reside in Malta and are covered by the Maltese social security legislation and/or have a valid working permit ${ }^{9}$. The OPAT service was introduced as an in-house infusion centre model in 2007 and extended to a home visiting nurse model in October 2016 with a view to introducing the self-administration model in the future. The latter model is supported by a multidisciplinary OPAT team of two doctors (one of which is the Head of Service), ten discharge liaison nurses and one pharmacist (the principal author). In addition, the OPAT team liaises with two infectious diseases consultant physicians and their assigned senior and junior doctors, the clinical pharmacist specialising in infectious diseases and one clerical staff member. Figure 1 illustrates the general framework for a patient episode receiving the OPAT service. 


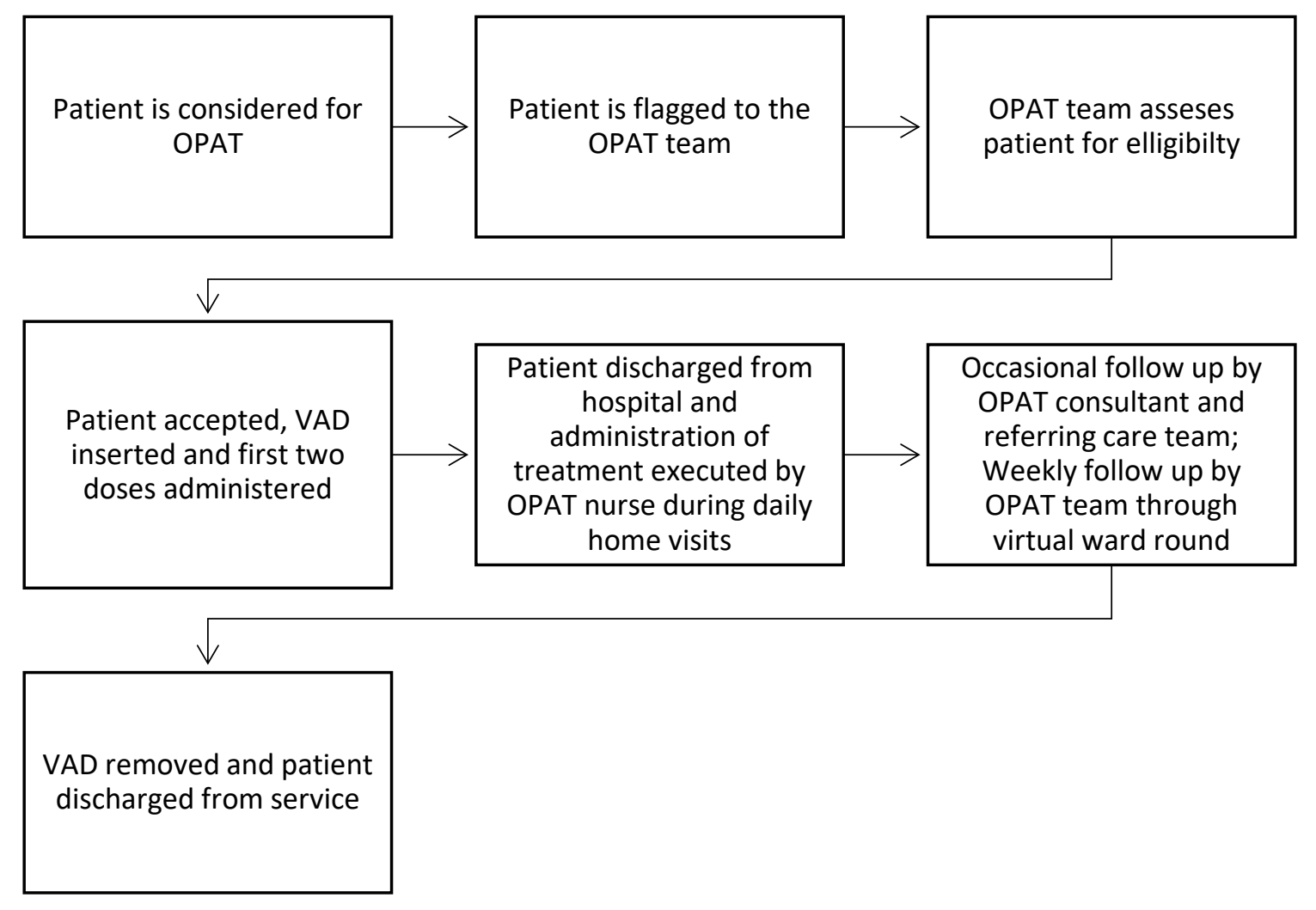

Figure 1 General framework for a patient episode receiving the OPAT service

(OPAT: Outpatient parenteral antimicrobial therapy; VAD: Vascular access device)

While there is extensive evidence to support the benefits of OPAT in terms of cost reductions, readmission rates and end user satisfaction ${ }^{3,5,10-12}$, there is a need to relate outcomes to the specific healthcare system under study. A systematic review by Boese et al. aimed to compare inpatient parenteral antibiotic therapy (IPAT) with OPAT. While specific to the orthopaedic context, the findings illustrated that differences in the complexity of healthcare systems, 
methods of reimbursement and the variation in daily costs for both inpatient stay and OPAT services limit generalisability to other settings ${ }^{13}$. Currently such data related to the local setting is not actively compiled and reported. To this aim, this study sought to evaluate the recently established Maltese visiting nurse OPAT service by determining service outcomes, centring on OPAT duration and OPAT completion status (improved, readmitted or deceased), and to estimate the costs required for service delivery using an activity-based approach.

\section{Methods}

\section{Design}

This study was an OPAT service evaluation from the perspective of the organisation.

\section{Setting}

The study was conducted at MDH, the acute general teaching hospital in Malta. $\mathrm{MDH}$ is the largest national public hospital in Malta, offering an array of specialist services in the fields of medicine, surgery, paediatrics, orthopaedics, cardiology, ophthalmology, neurology, dentistry and obstetrics and gynaecology. The services extend beyond the inpatient setting to include 
emergency, day care, outpatient and diagnostic services. MDH also houses three intensive care units, general, cardiac and paediatric.

Inclusion and exclusion criteria

All patients enrolled into the OPAT service from October 2016 to October 2019 were included, with no exclusions.

\section{Recruitment}

Patients were invited to participate in the study by the principal researcher, who provided each with a patient information leaflet.

\section{Data collection}

A database was created in Microsoft Excel ${ }^{\circledR}$, with the content reviewed by OPAT team members and piloted in ten patients prior to use. The data were collected prospectively, including patient variables (age, gender, referring care team, presenting infection), OPAT variables (antimicrobial and dosing regimen, vascular access device, duration) and OPAT completion status (improved, readmitted or deceased). These data were collected from electronic medical records and during ward-based discussions of patient progress. At the outset of each OPAT episode, the OPAT consultant was requested to forecast the anticipated duration and advise the type of vascular access device (VAD) best suited for that patient. 
Data analysis

Patient demographics (age, gender), OPAT episode characteristics (referring consultant, presenting infection, VAD and antimicrobial regimen) and OPAT completion status (improved, readmitted or deceased) were analysed descriptively. Patients making use of the service more than once were recorded as additional episodes since treatment courses and number of visits varied between episodes. If a readmission was not necessary, the patient was treated to the point of improvement.

The success rate of the service was deduced from the percentage of improved cases observed. A distinction in the nomenclature of completion status between 'improved' and 'cured' was not applicable to this study. This was mainly due to the diverse rationales supporting the referral of patients to the service and was thus subjective for each episode. Therefore an 'improved' status was assigned to those episodes which resulted in an organic cessation of the service and not a readmission. On the other hand, the failure rate was deduced from the readmission and deaths recorded. Given that there were no recorded deaths resulting from a shortcoming of the service, the failure rate was dependant on the percentage of readmitted episodes. 
Descriptive analysis was carried out on the duration of OPAT episodes (i.e. the number of days from the first to last OPAT nurse administration visit) per year, including the occurrence of multiple episodes over the three-year period. The distribution of the total observed duration was tested for normality using the Kolmogorov-Smirnov test. These data were compared to the duration forecast made by the OPAT consultant at the point of OPAT commencement (Wilcoxon signed-rank test, $p<0.05$ was considered statistically significant). The KruskalWallis test was used to determine the influence of categorical variables e.g. presenting infection on the observed duration of each episode. Considering more than one variable was found to be significant using the Kruskal-Wallis test, the collective impact of the significant variables (i.e. presenting infection, readmission status and VAD) was deduced using a generalised linear model (GLM). This form of linear regression caters for response variables which do not have normal distributions. The GLM, within the context of the national OPAT service, enabled the prediction of future OPAT episode durations based on the influence of these three significant variables.

As the observed duration had a right skewed distribution and did not satisfy the normality assumption, a gamma distribution and a reciprocal link function was 
used. Dummy coding was introduced to cater for the categorical variables namely presenting infection, VAD and occurrence of readmission. In the model, "I" represented the presenting infection, "V" represented the vascular access device, whilst " $R$ " represented the completion status. Since the model incorporated all the dummy variables, a value of 1 was considered if the claim was in that category whilst a value of 0 was considered if otherwise. This resultant model would serve as a template for predicting the durations of future episodes.

In the absence of detailed healthcare inpatient costs, an activity-based costing approach was carried out to calculate the weekly running cost of the service using the Consolidated Health Economic Evaluation Reporting Standards (CHEERS) checklist to guide the reporting style utilised. Considering OPAT team members carry out other functions in the hospital (not related to OPAT) the fulltime equivalent (FTE) was calculated for the nurses, doctors, clerk and pharmacist based on self-reported time allocations for their related duties. The FTE was calculated by dividing the summation of the employee's reported scheduled hours by the total of hours for a full-time work week (i.e. 40hours). When OPAT team members reported a range rather than a specific duration, minimum and maximum FTE units were calculated to offer a better reflection of 
the current situation. The cost incurred to employ each member to render OPAT tasks was calculated by multiplying the FTE by the mean salary (as stipulated by the public officers' salary scales published by the Maltese government) .

\section{Ethics Approval}

Ethical approval was attained from ethics committees affiliated to Robert Gordon University, United Kingdom (S137) and the University of Malta (FRECMDS_1819_004).

\section{Results}

Patient demographics and OPAT episode characteristics

Patient demographics and OPAT episode characteristics are given in Table 1. The mean age was 61.3 years ( \pm 14.9 , range $16-92)$ and 76 (65\%) were male. Patients were most commonly referred by infectious diseases' consultants ( $n=35,26.5 \%)$ followed by medical consultants ( $n=28,21.2 \%)$, vascular surgeons $(n=25,18.9 \%)$ and orthopaedic surgeons $(n=20,15.2 \%)$. The most common presenting infections were orthopaedic category (e.g. osteomyelitis, discitis, prosthetic joint infections; $n=66,50.0 \%)$, followed by abscesses $(n=24,18.2 \%)$, gastroenterology ( $n=11,8.3 \%)$ and cardiology $(n=10,7.6 \%)$. Almost all OPAT therapy ( $n=112,84.8 \%$ ) was delivered by the peripherally inserted central catheter (PICC). The most frequently prescribed antimicrobial regimen was 
ceftriaxone as a single agent $(n=52,34.9 \%)$. Seventeen (11.4\%) OPAT episodes required two antimicrobials with the most frequent combination being teicoplanin and ertapenem (Table 1).

\begin{tabular}{|l|l|}
\hline Number of patients (\%) (N=117) \\
\hline Male & $76(65.0)$ \\
Female & $41(35.0)$ \\
\hline Referring consultants for OPAT (\%) (N=132 episodes) \\
\hline Infectious Diseases & $35(26.5)$ \\
Medical & $28(21.2)$ \\
Vascular Surgeon & $25(18.9)$ \\
Orthopaedic Surgeon & $20(15.2)$ \\
General Surgeon & $9(6.8)$ \\
Cardiac & $5(3.8)$ \\
Ear, Nose, Throat (ENT) & $4(3.0)$ \\
Oncology & $3(2.3)$ \\
Urology & $3(2.3)$ \\
\hline Indications for OPAT (N=132 episodes) & \\
\hline Orthopaedic (e.g. discitis, prosthetic joint infection) & $66(50.0)$ \\
Abscess (e.g. liver, spinal, brain) & $24(18.2)$ \\
Gastroenterology (e.g. intra-abdominal infection) & $11(8.3)$ \\
Cardiology (e.g. infective endocarditis) & $10(7.6)$ \\
Oral and Respiratory Infections (e.g. bronchitis, COPD) & $8(6.1)$ \\
Bacteraemia & $7(5.3)$ \\
Nephrology (e.g. urinary tract infection) & $6(4.5)$ \\
\hline Type of OPAT VAD (N=132 episodes) & \\
\hline Peripherally inserted central catheter & $112(84.8)$ \\
Peripherally inserted intravenous cannula (Venflon $\left.{ }^{\circledR}\right)$ & $10(7.6)$ \\
Implantable venous access system & $7(5.3)$ \\
Peripherally inserted intravenous cannula (midline) & $3(2.3)$ \\
\hline Antimicrobial agents (N=149 agents) & $52(34.9)$ \\
\hline Ceftriaxone & $38(25.5)$ \\
Ertapenem & $21(14.1)$ \\
Teicoplanin & $19(12.8)$ \\
Ceftazidime & \\
\hline
\end{tabular}


Tigecycline

Meropenem

$8(5.4)$

$6(4)$

Colistimethate

$3(2)$

Piperacillin/tazobactam

$2(1.3)$

Combination antimicrobial courses $(\mathrm{N}=17)$

Teicoplanin and Ertapenem

Teicoplanin and Ceftriaxone

$9(52.9)$

Meropenem and Colistimethate

4 (23.5)

Ceftriaxone and Tigecycline

Ceftazidime and Colistimethate

$1(5.9)$

Table 1. Patient demographics and OPAT episode characteristics, $n$ (\%)

(OPAT: Outpatient parenteral antimicrobial therapy; VAD: Vascular access device; COPD: Chronic obstructive pulmonary disease)

Study outcome, OPAT duration

The number of OPAT episodes increased from 29 in the first year of the study (total of 736 OPAT days) to 47 in the second (1306 OPAT days) and 56 in the third (1245 OPAT days), giving 3287 OPAT days over the study timeframe. Data from 117 patients were collected throughout the three-year period; of these 15 patients (12.8\%) had two OPAT episodes. The median OPAT episode duration was 22 days (IQR 10.75-42). The observed OPAT duration data were not normally distributed (Kolmogorov-Smirnov, $p<0.01$ ), and were statistically significantly lower than the duration forecast by the OPAT consultant at the point of OPAT commencement (median 28 days, IQR 14-42; Wilcoxon signedrank test, $\mathrm{p}<0.01$ ) (Table 2 ). 
In univariate analysis, the following variables were statistically significantly in relation to OPAT duration: the type of VAD $(p<0.001)$; the presenting infection $(p=0.021)$; and the readmission rate $(p=0.005)$ (Kruskal-Wallis test).

The longest observed durations were mainly observed in patients who made use of a PICC line for drug administration (median=25 days, IQR=13-36). This result was followed by patients who had an implantable venous access system (median=18 days, IQR=10-23.5), peripherally inserted intravenous cannula midline (median=8 days, IQR=7.5-8) and Venflon ${ }^{\circledR}$ (median=6.5 days, IQR=57.75) respectively. As expected, longer observed durations were seen in those episodes which were not prematurely ceased due to a readmission (median=24 days, IQR=13-36) versus those with an episode which resulted in a readmission (median=11 days, IQR=7-25.5). In terms of presenting infections, longer durations were observed in the treatment of orthopaedic (median=29 days, IQR=15-35.75), abscess (median=21.5days, IQR=13-39.25), nephrology cases (median=21.5 days, IQR=8.25-46.75), oral and respiratory cases (median=21 days, IQR=7.75-29.5) and cardiology cases (median=19 days, IQR=13.25-23.5). On the other hand, shorter durations were observed in the treatment of gastroenterology cases (median=13 days, IQR=7-25) and bacteraemias (median=8 days, IQR=7-9). 
To take into consideration the simultaneous influence of these three significant variables, GLM was chosen. The coefficients generated deduced that all subparameters (dummy codes) pertaining to the presenting infection, readmission status and VAD influenced the final duration except for oral/respiratory infections, implantable venous access systems and episodes which did not require a readmission as evidenced by a value of zero. These sub-parameters were redundant in determining the duration of an episode and were thus removed. The following nomenclature was used for sub-parameters dummy codes: $I_{1}$ for orthopaedic cases, $I_{2}$ for cardiology cases, $I_{3}$ for gastroenterology cases, $I_{4}$ for abscess cases, $I_{5}$ for nephrology cases, $I_{6}$ for bacteraemia cases, $I_{7}$ for oral and respiratory cases; $R_{1}$ for readmission cases, $R_{2}$ for episodes which did not involve a readmission; $V_{1}$ for PICC lines, $V_{2}$ for midlines and $V_{3}$ for portacath and $V_{4}$ for implantable venous access systems. Since the exponential family function gamma was chosen for this analysis, a reciprocal link function was used. The intercept for this regression analysis was 0.086 and the deviance reflecting the goodness of fit was of $3.465(p=0.029)$. The GLM is the following: $1 /$ duration $=0.086+0.009 I_{1}+0.028 I_{2}+0.017 I_{3}+0.012 I_{4}+0.003 I_{5}+0.078 I_{6}+0.015 R_{1}-$ $0.062 \mathrm{~V}_{1}+0.037 \mathrm{~V}_{2}-0.052 \mathrm{~V}_{3}$

Study outcome, OPAT completion status (improved, readmitted or deceased) 
Of the 132 episodes, 109 resulted in an improved clinical status (i.e. provision of care without re-hospitalisation) giving a success rate of $82.6 \%$. The remaining 23 OPAT episodes were terminated due to readmission, 20 (87.0\%) of which were unplanned and 3 electives (13.0\%) (Table 2). 18 out of the 23 episodes were characterised by the utilisation of the PICC line as the patient's vascular access device (78.3\%). Unplanned admissions were largely for reasons of deterioration of presenting infection (e.g. fever, lethargy, diarrhoea, headaches) $(n=6,30.0 \%)$, worsening of symptoms relating to comorbidities $(n=3,15.0 \%)$ and erythema of infected sites ( $n=3,15.0 \%)$. Of the 20 unplanned readmission episodes, the majority were managing an orthopaedic related infection $(n=12,60 \%)$ followed by abscess cases $(n=3,15 \%)$. One OPAT patient died from oncology complications unrelated to OPAT provision.

\begin{tabular}{|l|l|}
\hline \multicolumn{2}{|l|}{ Episode completion status (N=132) } \\
\hline $\begin{array}{l}\text { Improvement } \\
\text { Readmitted from OPAT }\end{array}$ & $\begin{array}{l}109(82.6) \\
23(17.4)\end{array}$ \\
\hline Readmissions from OPAT (N=23) & $3(13.0)$ \\
\hline Elective & $20(87.0)$ \\
Unplanned & \\
\hline Unplanned Readmissions (N=20) & $6(30)$ \\
\hline Deterioration of presenting infection (e.g. fever, lethargy, & \\
diarrhoea, headaches) & $3(15)$ \\
Worsening symptoms of comorbidities & $3(15)$ \\
Erythema of infected site & $2(10)$ \\
Atrial Fibrillation & $2(10)$ \\
Deep vein thrombosis & $1(5)$ \\
Anaemia &
\end{tabular}


Social Factors

Death

Change to oral therapy

Table 2 Completion status following OPAT episodes, $\mathrm{n}(\%)$

(OPAT: Outpatient parenteral antimicrobial therapy)

Study outcome, costs required for service delivery from the perspectives of the organisation using an activity-based approach

Table 3 below indicates the OPAT related duties performed by the members of the team including the support staff. The three professional groups partook in two activities namely the virtual ward round and referrals. Other activities were specific to the team members to include administration outreaches for nurses, outpatient appointments for doctors and treatment preparation for the pharmacist.

\begin{tabular}{|l|l|l|l|}
\hline OPAT team member & Activity & $\begin{array}{l}\text { Minimum } \\
\text { duration }\end{array}$ & $\begin{array}{l}\text { Maximum } \\
\text { duration }\end{array}$ \\
\hline \multirow{2}{*}{ Nurse } & Treatment outreach & 1 hour & \\
\cline { 2 - 4 } & Virtual ward round & 1 hour/week & \\
\cline { 2 - 4 } & Referral & $30 \mathrm{mins}$ & $45 \mathrm{mins}$ \\
\hline \multirow{2}{*}{ Doctor } & Outpatient appointment & $15 \mathrm{mins}$ & \\
\cline { 2 - 4 } & Virtual ward round & 1 hour/week & \\
\cline { 2 - 4 } & Referral & $30 \mathrm{mins}$ & $45 \mathrm{mins}$ \\
\hline \multirow{2}{*}{ Pharmacist } & Treatment preparation & $20 \mathrm{mins}$ & \\
& Virtual ward round & 1 hour/week & \\
\cline { 2 - 4 } & Referral & $30 \mathrm{mins}$ & $45 \mathrm{mins}$ \\
\hline Clerk & Treatment collection & $30 \mathrm{mins}$ & \\
\hline
\end{tabular}

Table 3 Self-reported OPAT durations per task 
(OPAT: Outpatient parenteral antimicrobial therapy)

Table 4 shows the extrapolation of self-reported durations (Table 3) for the study timeframe and the FTE units assuming the national 40-hour full-time working week. The treatment outreach duration for each episode was calculated by multiplying the observed OPAT duration (the number of days from the first to last visit) by the number of daily visits. Since each outreach was estimated to last one hour, the value obtained reflected the treatment outreach duration. This calculation was performed for each of the 132 episodes. Based on the FTE units and the mean weekly salaries for 2019 , the mean relative salaries were the following €296.94/\$351.18, €24.38/\$29.54, €23.18/\$27.41 and €2.01/\$2.38 for nurses, doctors, pharmacists and clerks respectively. Thus, using these values and the calculated FTEs, the overall mean cost towards OPAT salaries equated to $€ 346.51 / \$ 409.81$. Table 4 also includes the cost of fuel consumption and car rental fee which was specific to the nurses' outreach activity. This data was gathered from the team's invoices rather than a self-reported duration as it was more accurate. 


\begin{tabular}{|c|c|c|c|}
\hline $\begin{array}{l}\text { OPAT } \\
\text { team/staff } \\
\text { member }\end{array}$ & Task description & Minimum & Maximum \\
\hline \multirow[t]{2}{*}{ Nurse } & $\begin{array}{l}\text { Treatment outreach } \\
\text { Referrals } \\
\text { Virtual weekly ward } \\
\text { rounds }\end{array}$ & $\begin{array}{l}4026 h \\
66 h \\
156 h\end{array}$ & $\begin{array}{l}4026 \mathrm{~h} \\
99 \mathrm{~h} \\
156 \mathrm{~h}\end{array}$ \\
\hline & $\begin{array}{l}\text { Total (over } 3 \text { years) } \\
\text { FTE } \\
\text { Mean nurse salary/week } \\
\text { Salary of nurse for OPAT } \\
\text { tasks }\end{array}$ & $\begin{array}{l}4248 \mathrm{~h} \\
0.68 \\
€ 434.50 / \$ 513.87 \\
€ 295.79 / \\
\$ 349.82 \\
\end{array}$ & $\begin{array}{l}281 \mathrm{~h} \\
0.69 \\
€ 298.09 / \\
\$ 352.55 \\
\end{array}$ \\
\hline \multirow[t]{2}{*}{ Doctor } & $\begin{array}{l}\text { Outpatient visit } \\
\text { Referrals } \\
\text { Virtual weekly ward } \\
\text { rounds }\end{array}$ & $\begin{array}{l}30 \mathrm{~h} \\
66 \mathrm{~h} \\
156 \mathrm{~h}\end{array}$ & $\begin{array}{l}30 h \\
99 h \\
156 h\end{array}$ \\
\hline & $\begin{array}{l}\text { Total (over } 3 \text { years) } \\
\text { FTE } \\
\text { Mean doctor salary/week } \\
\text { Salary of doctor for OPAT } \\
\text { tasks }\end{array}$ & $\begin{array}{l}252 \mathrm{~h} \\
0.04038 \\
€ 566.52 / \$ 670.01 \\
€ 22.88 / \$ 27.06\end{array}$ & $\begin{array}{l}285 h \\
0.04567 \\
€ 25.87 / \\
\$ 30.60\end{array}$ \\
\hline \multirow[t]{2}{*}{ Pharmacist } & $\begin{array}{l}\text { Treatment preparation } \\
\text { Referrals } \\
\text { Virtual weekly ward } \\
\text { rounds }\end{array}$ & $\begin{array}{l}44 h \\
66 h \\
156 h\end{array}$ & $\begin{array}{l}44 \mathrm{~h} \\
99 \mathrm{~h} \\
156 \mathrm{~h}\end{array}$ \\
\hline & $\begin{array}{l}\text { Total (over } 3 \text { years) } \\
\text { FTE } \\
\text { Mean pharmacist salary/ } \\
\text { week } \\
\text { Salary of nurse for OPAT } \\
\text { tasks }\end{array}$ & $\begin{array}{l}266 h \\
0.04263 \\
€ 511.88 / \\
\$ 605.39 \\
€ 21.82 / \\
\$ 25.81 \\
\end{array}$ & $\begin{array}{l}299 \mathrm{~h} \\
0.04791 \\
€ 24.53 / \\
\$ 29.01\end{array}$ \\
\hline \multirow[t]{2}{*}{ Clerk } & Treatment collection & $66 \mathrm{~h}$ & $\begin{array}{l}\text { As } \\
\text { minimum }\end{array}$ \\
\hline & $\begin{array}{l}\text { Total (over } 3 \text { years) } \\
\text { FTE }\end{array}$ & $\begin{array}{l}66 \mathrm{~h} \\
0.01058 \\
\end{array}$ & \\
\hline
\end{tabular}




\begin{tabular}{|l|l|l|l|} 
& $\begin{array}{l}\text { Mean clerk salary/ week } \\
\text { Salary of clerk for OPAT } \\
\text { tasks }\end{array}$ & $\begin{array}{l}€ 190.49 / 225.29 \\
€ 2.01 / \$ 2.38\end{array}$ & \\
\hline Travelling & Weekly consumption & $€ 26.92 /$ & $\begin{array}{l}\text { As } \\
\text { minimum }\end{array}$ \\
\cline { 2 - 4 } & Weekly car rental & $\$ 31.84$ & $\begin{array}{l}€ 82.05 / \\
\text { minimum }\end{array}$ \\
\hline Total weekly expenditure & $\$ 97.04$ & $€ 459.47 /$ \\
& $€ 451.47 /$ & $\$ 543.41$ \\
\hline Average weekly expenditure & $\$ 533.94$ & \\
\hline Total daily expenditure & $€ 455.47 /$ & \\
& $\$ 538.68$ & \\
\hline
\end{tabular}

Table 4. Duration (minimum and maximum) of general tasks performed by OPAT team over the three-year period and the FTE units for each member of staff (OPAT: Outpatient parenteral antimicrobial therapy; FTE: Full time equivalent)

\section{Discussion}

After three years, the service resulted in 3287 bed days saved through the provision of OPAT. These results are based on the 132 OPAT episodes undertaken by the OPAT team employed by MDH. Moreover, a total of 23 episodes were prematurely terminated due to a readmission thus resulting in a readmission rate of $17.4 \%$ and a success rate of $82.6 \%$. By means of an activitybased costing exercise, it was deduced that it would cost the institution a mean of $€ 455.47 / \$ 538.68$ per week to cover the expenses of the service provision.

Given that OPAT is an extension of services offered by the local hospital, the patients did not incur any fees, thus enabling evidence-based prescribing 
without financial constraints ${ }^{9}$. This study's findings corroborate results published about other OPAT practices including the abundant use of Ceftriaxone $e^{10,14}$, simultaneous antimicrobial agent administrations ${ }^{1,15}$ and frequent insertions of PICC lines ${ }^{1,16}$.

The primary measurable outcome of this study was the observed duration of the patient's OPAT episode since it had a direct impact on the institution's bed occupancy. Despite being shorter than that forecasted by the OPAT clinicians $(p<0.01)$, statistical analysis deduced that the presenting infection $(p=0.021)$, $\operatorname{VAD}(p<0.001)$ and the occurrence of a readmission $(p=0.005)$ had a significant influence. These variables were used to give rise to one of the most significant contributions made by this study i.e. the design of a predictor model to forecast the duration of future OPAT episodes. The ability to make predictions about a patient's OPAT journey implies that from the onset, the OPAT team can estimate the resources needed to successfully treat a patient. Even though the occurrence of a readmission is unknown (since present clinical instability would not make a patient eligible for OPAT), the model could be utilised to generate a timeframe using both eventualities i.e. one in which a readmission took place and one where the patient was successfully treated through OPAT.

In the systematic review by Sriskandarajah's et al., OPAT studies reported success rates to be greater than $80 \%$, readmission rates between $1 \%$ and $14.3 \%$ 
and mortality rates between $0 \%$ and $1.4 \%{ }^{17}$. With a local success rate of $82.6 \%$, and a $17.4 \%$ readmission rate, the Maltese service is at par with other services reported in the literature $\mathrm{e}^{1,7,18}$.

A cost-benefit is often stated as a desirable aspect of OPAT, but there is often little clarity and comparability due to the different reporting styles and available data to generate economic outcomes ${ }^{19}$. To quantify the relative salary required to perform OPAT staff duties, the concept of FTE units was adapted from a study published by Wai et al. ${ }^{20}$. This was incorporated in an activity-based costing exercise which was crucial in establishing a budget for the service, previously unknown to the institution. The mean cost to run the service daily ranged between $€ 64.50 / \$ 76.28$ and $€ 65.64 / \$ 77.63$ which is comparable to costings reported in the literature ${ }^{13}$. Similar approaches have been used to establish the cost of resources to provide OPAT to include cost-consequence analysis ${ }^{7}$ and resource use top down strategy ${ }^{21}$.

However, there are certain assumptions that must be considered in terms of the costing exercise carried out in this study. Considering MDH provides both IPAT and OPAT, the cost of the VAD insertion was not considered since this activity would have taken place irrespective of the setting. Moreover, the employment costs incurred by the institution were deduced from the salary brackets based 
on the grades of the current OPAT team and the corresponding annual pay for the year 2019, excluding allowances. Using basic salaries in this way is likely to underestimate the full economic costs, and is therefore a limitation of the study.

Despite these assumptions, it is the first prospective study to benchmark the Maltese visiting nurse OPAT model since its inception in 2016. The specificity of hospitalisation costs to the location of the ward bed (general, high dependency or intensive care), impeded the quantification of the cost of IPAT. This in turn made it difficult to make a comparison between IPAT and OPAT which would in turn deduce the financial burden saved to the institution by providing this service. Apart from investigating the later costing exercise, it would be of interest to apply the activity-based approach to other hospital services considering the absence of robust healthcare fees locally. In addition, future research can investigate the resolution of the readmitted OPAT cases e.g. required a hospital stay, discharged and switched to oral therapy, referred to the OPAT infusion centre or reinstated in the OPAT service.

\section{Conclusion}

The number of successfully completed episodes demonstrates the achievement of the Maltese visiting nurse OPAT model. From the patient's clinical 
improvement and the low readmission rates, it is evident that the service has reduced bed occupancy without compromising the standard quality of care. In clearly defining the outcome measures and the cost of the service, this work addresses some of the issues in the literature around clarity of reporting. In conclusion, this study sheds light on the need for standardisation in measuring incurred costs which would in turn lead to a more robust cost analysis.

Acknowledgments: The authors wish to thank the dedicated OPAT team comprising infectious diseases physicians and nurses for their sterling service. Moreover, a special thanks to Dr. Denise Sammut Alessi and Dr. Neil McEwan for their contribution to this paper.

Declaration of interest: None

Funding: This research did not receive any specific grant from funding agencies in the public, commercial, or not-for-profit sectors.

Ethical approval: this study was approved by the ethics committees affiliated to Robert Gordon University, United Kingdom and University of Malta in partial fulfilment of the first author's doctoral degree.

\section{References}

1. Norris AH, Shrestha NK, Allison GM, Keller SC, Bhavan KP, Zurlo JJ et al. 2018 Infectious Diseases Society of America clinical practice guideline for the 
management of outpatient parenteral antimicrobial therapy. Clin. Infect. Dis 2019;68(1): e1-35.

2. Rucker RW, Harrison GM. Outpatient intravenous medications in the management of cystic fibrosis. Pediatrics 1974; 54(3):358-60.

3. Durojaiye OC, Cartwright K, Ntziora F. Outpatient parenteral antimicrobial therapy (OPAT) in the UK: a cross-sectional survey of acute hospital trusts and health boards. Diagn Micr Infec Dis 2019; 93(1):58-62.

4. Wee LE, Sundarajoo M, Quah WF, Farhati A, Huang JY, Chua YY. Sociodemographic and clinical factors associated with acceptance of outpatient parenteral antibiotic therapy in a Singapore tertiary hospital from 2014 to 2017. Eur J Clin Microbiol 2019;38(2):277-84.

5. Mansour O, Heslin J, Townsend JL. Impact of the implementation of a nursemanaged outpatient parenteral antibiotic therapy (OPAT) system in Baltimore: a case study demonstrating cost savings and reduction in re-admission rates. J Antimicrob Chemo 2018;73(11):3181-8.

6. Petrak RM, Skorodin NC, Fliegelman RM, Hines DW, Chundi VV, Harting BP. Value and clinical impact of an infectious disease-supervised outpatient parenteral antibiotic therapy program. Open Forum Infect Dis 2016;1(4) 
7. Durojaiye OC, Bell H, Andrews D, Ntziora F, Cartwright K. Clinical efficacy, cost analysis and patient acceptability of outpatient parenteral antibiotic therapy (OPAT): a decade of Sheffield (UK) OPAT service. Int J Antimicrob Agents 2018; 51(1):26-32

8. Chapman AL, Patel S, Horner C, Green H, Guleri A, Hedderwick S et al. Updated good practice recommendations for outpatient parenteral antimicrobial therapy (OPAT) in adults and children in the UK. JAC-AMR 2019;1(2):dlz026.

9. Azzopardi Muscat N, Calleja N, Buttigieg S, Merkur S. Malta: health system review. Health Syst Transit 2017;19(1):1-37.

10. Minton J, Murray CC, Meads D, Hess S, Vargas-Palacios A, Mitchell E, et al. The Community Intravenous Antibiotic Study (CIVAS): a mixed-methods evaluation of patient preferences for and cost-effectiveness of different service models for delivering outpatient parenteral antimicrobial therapy. Health Serv Deliv Res 2017;5(6).

11. Twiddy M, Czoski Murray CJ, Mason SJ, Meads D, Wright JM, Mitchell ED, et al. A qualitative study of patients' feedback about Outpatient Parenteral Antimicrobial Therapy (OPAT) services in Northern England: implications for service improvement. BMJ Open 2018; 8(1):e019099 
12. Shah A, Petrak R, Fliegelman R, Shrestha N, Allison G, Zurlo J et al. Infectious diseases specialty intervention is associated with better outcomes among privately insured individuals receiving outpatient parenteral antimicrobial therapy. Clin. Infect. Dis 2019;68(7):1160-5.

13. Boese CK, Lechler P, Frink M, Hackl M, Eysel P, Ries C. Cost-analysis of inpatient and outpatient parenteral antimicrobial therapy in orthopedics: A systematic literature review. WORLD J CLIN CASES 2019; 7 (14): 1825.

14. Yang C, Nguyen NN, Chary A, Nakasone TS. Outpatient Parenteral Antimicrobial Therapy (OPAT) Practices at a Veterans Affairs Hospital: Potential for Pharmacist Impact. Open Forum Infect Dis 2017; 4(Suppl 1):S331 15. Tan SJ, Ingram PR, Rothnie AJ, Whitmore TJ, Robinson JO, Hatch JB, et al. Successful outpatient parenteral antibiotic therapy delivery via telemedicine. J Antimicrob Chemother 2017;72(10):2898-2901

16. Keller SC, Dzintars K, Gorski LA, Williams D, Cosgrove SE. Antimicrobial Agents and Catheter Complications in Outpatient Parenteral Antimicrobial Therapy. Pharmacotherapy 2018;38(4):476-481.

17. Sriskandarajah S, Hobbs J, Roughead E, Ryan M, Reynolds K. Safety and effectiveness of 'hospital in the home' and 'outpatient parenteral 
antimicrobial therapy' in different age groups: A systematic review of observational studies. Int J Clin Pract 2018: e13216.

18. Bellamy R. Outpatient parenteral antimicrobial therapy. BRIT J HOSP MED 2018;79(1):12-7.

19. MacKenzie M, Rae N, Nathwani D. Outcomes from global adult outpatient parenteral antimicrobial therapy programmes: a review of the last decade. Int. J. Antimicrob. Agents 2014;43(1):7-16.

20. Wai AO, Frighetto L, Marra CA, Chan E, Jewesson PJ. Cost Analysis of an Adult Outpatient Parenteral Antibiotic Therapy (OPAT) Programme. Pharmacoeconomic 2012; 18, 451-457.

21. González-Ramallo VJ, Mirón-Rubio M, Mujal A, Estrada O, Forné C, Aragón B et al. Costs of outpatient parenteral antimicrobial therapy (OPAT) administered by Hospital at Home units in Spain. Int. J. Antimicrob. Agents 2017;50(1):114-8. 